\title{
Care coordination between specialty care and primary care: a focus group study of provider perspectives on strong practices and improvement opportunities
}

\author{
This article was published in the following Dove Press journal: \\ Journal of Multidisciplinary Healthcare \\ 22 January 2015 \\ Number of times this article has been viewed
}

\author{
Bo Kim ${ }^{1,2}$ \\ Michelle A Lucatorto ${ }^{3}$ \\ Kara Hawthorne ${ }^{4}$ \\ Janis Hersh ${ }^{5}$ \\ Raquel Myers ${ }^{6}$ \\ A Rani Elwy ${ }^{1,7}$ \\ Glenn D Graham ${ }^{8}$ \\ 'Center for Healthcare Organization \\ and Implementation Research, Edith \\ Nourse Rogers Memorial VA Hospital, \\ Bedford, ${ }^{2}$ Department of Psychiatry, \\ Harvard Medical School, Boston, \\ MA, ${ }^{3}$ Office of Nursing Services, \\ Department of Veterans Affairs, \\ ${ }^{4}$ Chief Business Office, Purchased \\ Care, Washington, DC, ${ }^{5} \mathrm{New}$ England \\ Veterans Engineering Resource \\ Center, Boston, MA, ${ }^{6} \mathrm{~S}$ U Quinney \\ College of Law, University of Utah, \\ Salt Lake City, UT, ' Department \\ of Health Policy and Management, \\ Boston University School of Public \\ Health, Boston, MA, ${ }^{8}$ Specialty Care \\ Services (IOP4E), Department of \\ Veterans Affairs, Washington, DC, USA
}

Correspondence: Bo Kim Center for Healthcare Organization and Implementation Research, Edith Nourse Rogers Memorial VA Hospital, 200 Springs Road (I52), Bedford, MA 01730, USA

$\mathrm{Tel}+\mathrm{I} 78 \mathrm{I} 68734 \mathrm{I2}$

Fax + I 7816873106

Email bo.kim@va.gov
Abstract: Care coordination between the specialty care provider (SCP) and the primary care provider (PCP) is a critical component of safe, efficient, and patient-centered care. Veterans Health Administration conducted a series of focus groups of providers, from specialty care and primary care clinics at VA Medical Centers nationally, to assess 1) what SCPs and PCPs perceive to be current practices that enable or hinder effective care coordination with one another and 2) how these perceptions differ between the two groups of providers. A qualitative thematic analysis of the gathered data validates previous studies that identify communication as being an important enabler of coordination, and uncovers relationship building between specialty care and primary care (particularly through both formal and informal relationship-building opportunities such as collaborative seminars and shared lunch space, respectively) to be the most notable facilitator of effective communication between the two sides. Results from this study suggest concrete next steps that medical facilities can take to improve care coordination, using as their basis the mutual understanding and respect developed between SCPs and PCPs through relationship-building efforts.

Keywords: referral and consultation, interdepartmental relations, multidisciplinary communication, qualitative research

\section{Introduction}

Failure of specialty care (SC) and primary care (PC) services in providing coordinated care can result in major delays in diagnosis, iatrogenic complications, and even mortality. ${ }^{1}$ Effective communication between the specialty care provider (SCP) and the primary care provider (PCP) has long been seen as a key requirement for successful coordination, and studies continue to identify communication breakdowns as the main cause for unclear expectations of roles and responsibilities between providers. ${ }^{2-5}$

In recent years, quality improvement and systems redesign work have been implemented and examined the effects of strategies to improve communication between the SCP and the PCP, with mixed results. Using a shared electronic health record (EHR) system increased access to information, but induced frustration when data were not made available to providers in a structured manner. ${ }^{6}$ Also, exchanging direct feedback with one another was appreciated by providers, but caused distress in some cases due to the increased workload. ${ }^{7}$ Communication mechanisms by themselves were not enough; the mechanisms needed to be designed with careful considerations given to 
what information to transfer using them, as well as how practical they are for both the SCP and the PCP.

This study, conducted by Veterans Health Administration (VHA) at representative medical facilities across all of its regional networks in the USA (called Veterans Integrated Services Networks [VISNs]), focuses on answering two main questions: 1) What do SCPs and PCPs perceive to be current practices that enable or hinder effective care coordination with one another? 2) How do these perceptions differ between SCPs and PCPs? Care coordination, for which no consensus definition yet exists, is broadly considered by the Agency for Healthcare Research and Quality (AHRQ) to include any mechanism that helps connect information, resources, and preferences of stakeholders involved in the care of a patient (ie, patient/family, health care professionals(s), and system representative(s), as categorized by AHRQ) ${ }^{8}$ We adopt here this broad definition in examining the coordination between SCPs and PCPs.

Noting the limited availability of established conceptual frameworks by which to study care coordination in health care systems, AHRQ's 2007 report on care coordination ${ }^{9}$ consolidates four separate yet relevant frameworks - the Andersen Behavior Framework, ${ }^{10}$ Donabedian's Quality Framework, ${ }^{11,12}$ the Organizational Design Framework, ${ }^{13}$ and the Relational Coordination Framework ${ }^{14}$ - into one operational summary of concepts across them all (Figure 1). Of the three areas into which the summary framework categorizes care coordination concepts, our work focuses on coordination between $\mathrm{SC}$ and $\mathrm{PC}$ regarding the first two areas, baseline assessment and coordinating mechanisms, in order to determine current strong practices and improvement opportunities that can lead to desirable changes in the third area of outcomes. Baseline assessment consists of a) need for coordination, b) predisposing characteristics, c) enabling resources, d) structures of care, and e) information requirements, which are concepts that frame the current state of coordination; coordinating mechanisms consist of a) relational coordination, b) organizational design options, and c) coordination processes, which are concepts that frame interventions that can be made to improve coordination.

We demonstrate the use of focus groups followed by thematic analysis ${ }^{15}$ as an approach to gathering and analyzing data that can evaluate care coordination in terms of the concepts under the two areas, which is essential for subsequently planning studies to uncover the two areas' effects on the third area of outcomes. Applied here to VHA's network of medical facilities, the approach is applicable to investigating any health care system for its care coordination between SC and PC.

\section{Methods}

\section{Focus group design}

Between May and October of 2011, a focus group of SCPs and another of PCPs were conducted at 24 of VHA's health care facilities across the nation (for a total of 48 focus group sessions); at least one facility from each of the 21 VISNs was included - 14 large, tertiary care medical centers and ten smaller facilities that deliver less extensive clinical services. This study belonged to a series of improvement projects

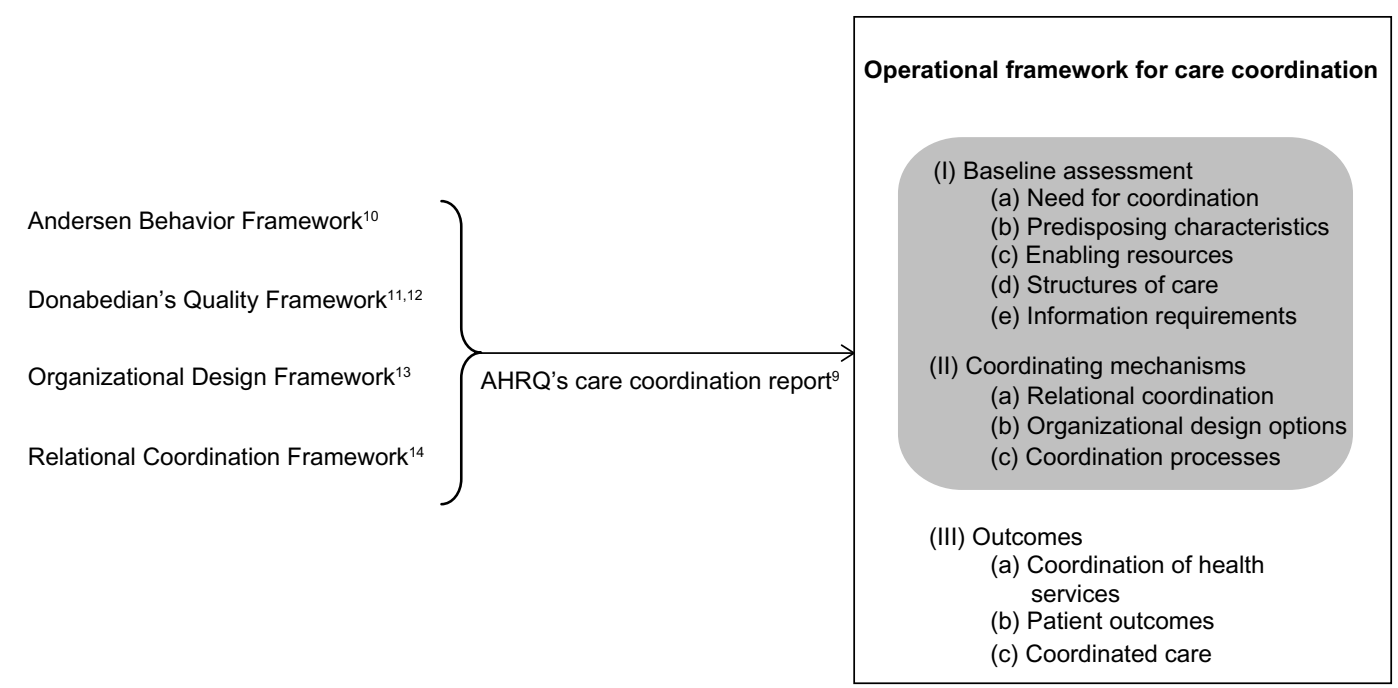

Figure I Agency for Healthcare Research and Quality (AHRQ)'s 2007 report on care coordination' consolidates four relevant frameworks into one operational summary framework, which categorizes concepts into three areas - (I) baseline assessment, (II) coordinating mechanisms, and (III) outcomes; our work demonstrates the use of focus groups and thematic analysis to examine I and II. 
classified to be not constituting research as described in VHA Handbook 1058.05 (and in turn exempt from human subjects review) by the Improvement Resources Office in VISN 1 (New England Healthcare System). ${ }^{16}$

Participants included physicians as well as nurse practitioners and physician assistants, and were recruited at individual facilities by the facility Director and the Chief of Staff; participation was voluntary, and no record of their attendance was reported back to their supervisors. Although separate focus group sessions were scheduled for SCPs and PCPs at each facility, participants who could not attend their originally assigned session due to time constraints were encouraged to attend the other. Also, providers at community clinics associated with the facilities were encouraged to participate in sessions over the telephone.

Each focus group session was facilitated by moderators trained by VHA's National Center for Organization Development. Fourteen moderators were trained in March and April of 2011 on facilitating the sessions and noting participant responses, as well as on the intent of the study and the planned use for the data to be collected. The trained moderators generally had backgrounds in education, social work, or psychology within VHA, selected for their familiarity with interpersonal processes and experience in facilitating groups to effectively elicit opinions from all participants. Each session lasted approximately 2 hours, during which one moderator asked the entire group a standard list of openended questions addressing the participants' thoughts on the interaction between SC and PC at their facility:

- Considering your experiences interacting with SCPs/ PCPs in your facility, what is working well in the communication and referral process?

- What are the barriers to good communication with SCPs/ PCPs at your facility?

- What are the problems with the referral/consultation process?

- What are your suggestions to improve the referral process between PC and SC?

- What other frustrations (beyond communication) do you experience in working with SCPs/PCPs at your facility?

- What other suggestions do you have to improve communications and operations between PC and SC?

- What is your perspective on how patients feel about their interaction with SC/PC? What do you hear from them?

- Have you developed strong relationships with one or more SCPs/PCPs? If so, how did it happen and how has it affected communications and/or referral processes?
This set of questions was developed by VHA's Office of Specialty Care Transformation, which originally launched this study to understand the state of SC-PC coordination in preparation for its initiatives to improve how SC is delivered within VHA (eg, a nationwide learning collaborative ${ }^{17}$ to redesign $\mathrm{SC}$ practices for enhanced access and care coordination).

The participants' responses were simultaneously scribed by a second moderator in the style of field notes, leaving out names, positions, and other subject identifiers. No audio recorders were used in collecting these responses, and participants were assured of and requested to uphold confidentiality regarding any remarks exchanged during the meeting.

\section{Thematic data analysis}

The scribed participant responses were analyzed through a thematic coding approach. ${ }^{15,18}$ Initially, three analysts reviewed every scribed response from focus group meetings held at four of the 24 facilities. These four (from four different VISNs; two large tertiary care medical centers and two smaller facilities that deliver less extensive clinical services) were the first facilities from which scribed responses had become available to the analysts. The analysts each independently created a list of themes that was believed to encompass all the responses reviewed, and the three lists were consolidated into an initial draft of a theme index. This index was continuously revised until analysis was completed on scribed responses from all 24 facilities, as described in the following paragraphs of this section.

Each of the 2,413 responses from all the focus group meetings was coded with a theme listed in the theme index. With two additional analysts contributing to the study, the coding was distributed evenly among five analysts. Each response was first assigned a theme by one analyst, and then independently assigned a theme by a second analyst; if the two assignments matched, the response was coded with that matching theme. Otherwise, the second analyst was responsible for deciding whether the first assigned, the second assigned, or another theme from the index best represents the response.

Each analyst's contribution to the coding task was divided evenly between serving as the first analyst on a subset of responses and as the second analyst on another, increasing the objectivity of the thematic coding approach. Furthermore, varied combinations of two out of the five analysts made up the pairs of first analyst and second analyst working together to assign themes to particular subsets of responses. All coding activity was recorded and analyzed using Excel spreadsheet software (Microsoft Corporation, Redmond, WA, USA). 
The theme index was actively revised throughout the process of assigning themes to responses from all the focus group meetings, mainly to accommodate for new themes that arose beyond the ones from the initial four facilities reviewed. A total of 86 themes were identified, and the five analysts who performed the coding of scribed responses held multiple discussions to reach a consensus on seven topics into which the themes were categorized:

- Access (themes related to access for patient appointments and availability to reach $\mathrm{SCPs} / \mathrm{PCPs}$ on the phone)

- Communication (themes related to relationships, training, and communication between SCP and PCP teams)

- Infrastructure (themes related to geographical distances, outpatient clinics, space, and information systems and technology)

- Patients (themes related to patient perceptions, quality of care for patients, and patient characteristics)

- Roles Clarity (themes related to organizational roles [ie, who is responsible for what])

- Tasks Structure (themes related to task guidelines and standards [ie, what is expected to be done])

- Workload (themes related to supply/staffing and workload balance)

In addition to being categorized into the seven topics, the themes were also classified in terms of their nature as either a "Strong Practice" theme, representing responses about existing practices that work well, or an "Improvement Opportunity" theme, representing responses about current practices that can be made better. This classification was carried out with advice from program officers at VISN1's Improvement Resources Office, who are experts in identifying gaps between observed and exemplary health care practices.

\section{Results}

We examined our thematically coded data in terms of the frequency with which each theme was mentioned at the focus group sessions. Recognizing the possibility that not using audio recorders may have resulted in some responses being accidentally not recorded, we also assessed each theme in terms of the number of facilities at which it was mentioned. We next discuss our findings regarding the nature of responses and recurring themes.

\section{Nature of responses}

Approximately $26.5 \%$ of all the responses reviewed relate to currently satisfactory practices, while the remaining $73.5 \%$ of responses relate to practices that are currently less satisfactory or ideas for improving current practices. For example, "interdisciplinary meetings help" is a Strong Practice theme about participants finding such meetings meaningful, which shows how events that foster interactions between providers can serve as a useful mechanism for relational coordination, as outlined in the conceptual framework (Figure 1, II.a). In contrast, "noneffective service/coordination agreements" is an Improvement Opportunity theme about existing agreements not working well, which shows the need for coordination beyond where it stands at the current baseline (Figure 1, I.a), chiefly in terms of better defining responsibilities for each provider involved in delivering the care.

Participants shared their thoughts on both strong practices and improvement opportunities at each facility. There are no notable differences between the responses from tertiary care medical centers and those from smaller facilities, and no particular subset of facilities is responsible for predominantly sharing strong practices versus improvement opportunities. Also, responses in most topic areas cover both strong practices and improvement opportunities.

\section{Recurring themes}

Searching for the tightest subset of themes that could account for approximately $20 \%$ of all the responses reviewed led to the five most frequently mentioned themes, shown in Table 1 ,

Table I Top five themes by number of times mentioned

\begin{tabular}{|c|c|c|c|c|}
\hline Theme & Number of responses & Topic & Nature of response & $\begin{array}{l}\text { Primary associated concept } \\
\text { in operational framework for } \\
\text { care coordination }\end{array}$ \\
\hline $\begin{array}{l}\text { Building relationships improves } \\
\text { the work }\end{array}$ & 120 & Communication & Strong Practice & II.a (relational coordination) \\
\hline Lack of care coordination & 91 & Patients & Improvement Opportunity & I.a (need for coordination) \\
\hline $\begin{array}{l}\text { Ineffective/incorrect/missing } \\
\text { templates for consult request } \\
\text { by PCP }\end{array}$ & 90 & Tasks structure & Improvement Opportunity & I.c (enabling resources) \\
\hline Lack of feedback & 80 & Communication & Improvement Opportunity & I.e (information requirements) \\
\hline Difficulty contacting SCP team & 77 & Communication & Improvement Opportunity & II.c (coordination processes) \\
\hline Top 5 of 86 themes & $19 \%$ of 2,413 responses & & & \\
\hline
\end{tabular}

Abbreviations: $\mathrm{PCP}$, primary care provider; SCP, specialty care provider. 
along with their primary associated concepts in the framework for care coordination (Figure 1). Four of these top five are Improvement Opportunity themes (as opposed to Strong Practice themes), which indicate the need for improved infrastructure or mechanisms to transfer information between $\mathrm{SC}$ and PC clinics. The number of responses for all themes, organized by topic, is provided as the Supplementary material (Table S1-S7).

The theme "building relationships improves the work" was mentioned more frequently than any other theme that arose throughout all the focus group meetings, reflecting how relationships that build over time between SCPs and PCPs are considered to be extremely valuable by the providers. Study participants mentioned both formal relationship-building events (eg, seminars led by SCPs for PCPs on specialty topics, and vice versa) and informal opportunities for interaction (eg, shared lunch space) being important practices. One provider stated,

I think when there is regular contact between a specialist and primary care provider, communication is improved. When they have met you professionally, there is improved communication and it builds relationships. In outlying clinics some specialists have come to visit and meet the staff.

The next two most frequently mentioned themes, "lack of care coordination" and "ineffective/incorrect/missing templates for consult request by PCP," both point to the common lack of straightforward guidelines as to how and what information to share between SCPs and PCPs for effective care coordination. One provider stated that "care is not coordinated," often requiring patients to make "multiple visits and travel." And another said, "There is miscommunication about whether or not a patient should go back to PCP or specialty for follow-up appointment."
Searching for themes that were mentioned at all but five or less of the participating facilities led to the ten themes that were mentioned at the greatest number of different facilities, shown in Table 2, along with their primary associated concepts in the framework for care coordination (Figure 1). The theme "informal consults are appreciated" was mentioned at as many facilities as the most frequently mentioned themes, emphasizing the value providers place on personal relationships and just-in-time information for care coordination. One provider stated, "Having an avenue where you can reach someone by phone has been extremely helpful," and another said, "Discussions with residents sometimes provide guidance that helps to avoid an unnecessary specialty visit," which shows how more appropriate care can be provided to the patient through such coordination built on relationships between the providers.

Also mentioned at many facilities were the themes "gaps in communication with patient" and "emphasis on communicating with patients," indicating the importance of patient communication in effective care coordination. One provider stated, "Patients constantly complain that they aren't being notified of specialty appointments"; another recommended an "after-visit summary" be "provided to the patient by specialty care" in order to facilitate a more coordinated transition back to $\mathrm{PC}$ for the patient.

Although flexibility for the attendees' schedules created mixed focus groups of both SCPs and PCPs in a small number of cases (specifically, a PCP being present in an SC focus group was noted by the session moderator in two instances, and an SCP being present in a PC focus group was noted by the session moderator in one instance; the study analysts carefully reviewed scribed responses from these focus groups to reach agreement that no hesitations toward open discussion were more detectable from these mixed groups' responses than from

Table 2 Top ten themes by number of facilities at which they were mentioned

\begin{tabular}{|c|c|c|c|c|}
\hline Theme & $\begin{array}{l}\text { Number } \\
\text { of facilities }\end{array}$ & Topic & Nature of response & $\begin{array}{l}\text { Primary associated concept in } \\
\text { operational framework for care } \\
\text { coordination } 9\end{array}$ \\
\hline Building relationships improves the work & 22 & Communication & Strong Practice & Il.a (relational coordination) \\
\hline Lack of care coordination & 22 & Patients & Improvement Opportunity & I.a (need for coordination) \\
\hline Informal consults are appreciated & 22 & Communication & Strong Practice & II.c (coordination processes) \\
\hline $\begin{array}{l}\text { Ineffective/incorrect/missing templates for } \\
\text { consult request by PCP }\end{array}$ & 21 & Tasks structure & Improvement Opportunity & I.c (enabling resources) \\
\hline Gaps in communication with patient & 21 & Patients & Improvement Opportunity & I.e (information requirements) \\
\hline Lack of feedback & 20 & Communication & Improvement Opportunity & I.e (information requirements) \\
\hline Emphasis on communicating with patients & 20 & Patients & Strong Practice & I.d (structures of care) \\
\hline Noneffective service/coordination agreements & 20 & Roles clarity & Improvement Opportunity & I.a (need for coordination) \\
\hline Interdisciplinary meetings help & 20 & Communication & Strong Practice & Il.a (relational coordination) \\
\hline Difficulty contacting SCP team & 19 & Communication & Improvement Opportunity & II.c (coordination processes) \\
\hline
\end{tabular}

Abbreviations: PCP, primary care provider; SCP, specialty care provider. 


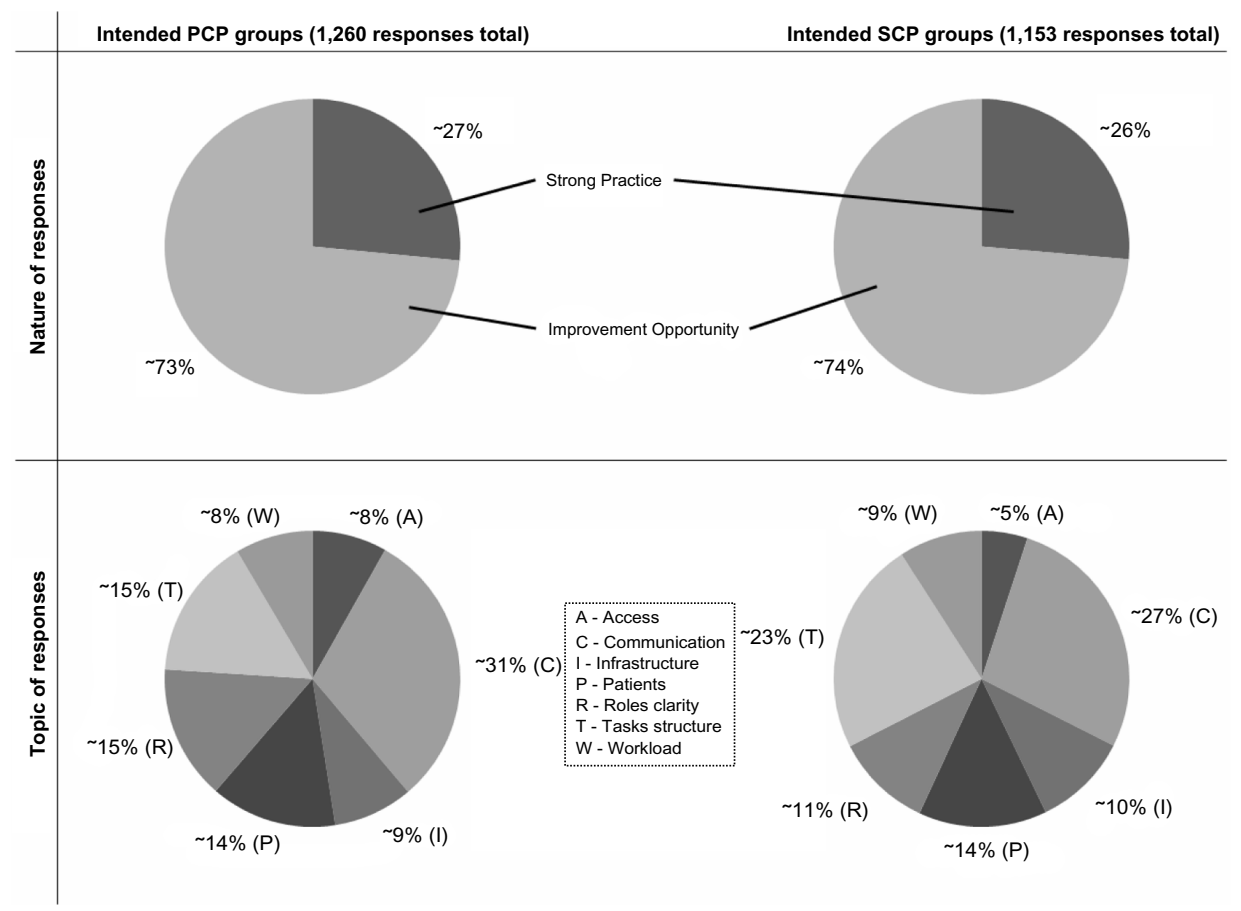

Figure 2 Nature and topic of responses mentioned at intended PCP groups and at intended SCP groups.

Abbreviations: PCP, primary care provider; SCP, specialty care provider.

other groups' responses, leading to our decision to not disregard valuable collected data from these groups in our analysis), data presented in this section can be additionally dissected to compare responses gathered from intended SCP groups alongside those gathered from intended PCP groups (Figure 2). Responses from the intended SCP and intended PCP groups had about the same proportion of Strong Practice responses (approximately $26 \%$ and $27 \%$, respectively) and Improvement Opportunity responses (approximately $74 \%$ and $73 \%$, respectively).

Responses related to Access made up a larger proportion for the intended PCP groups $(\sim 8 \%)$ than for the intended SCP groups $(\sim 5 \%)$, accounted for by the prevalence of the theme "poor SCP access for patients" among the PCP groups" responses. One PCP stated that there is "no uniformity in terms of clinic times" during which SCPs are available, and another recommended "allowing more direct contact between the patients and specialty care."

Responses related to Communication also made up a larger proportion for the intended PCP groups $(\sim 31 \%)$ than for the intended SCP groups ( 27\%), accounted for by the prevalence of the theme "lack of feedback" among the PCP groups' responses. Particularly with regard to consult requests submitted to SC, one PCP stated, "There needs to be more clarity about whether or not a new consult is needed. It can be redundant work," and another said that "discontinuation of consults without notification to the referring physician and no follow-up appointments" is problematic.
Responses related to Roles Clarity made up a larger proportion for the intended PCP groups ( $\sim 15 \%)$ than for the intended SCP groups $(\sim 11 \%)$ as well, accounted for by the prevalence of the theme "noneffective service/coordination agreements" among the PCP groups' responses. One PCP mentioned being "not satisfied with the service level agreements that are embedded in the consult request," and also stated, "Requirements are often arbitrary and there is a lot of extra typing, which is time-consuming."

On the other hand, responses related to Tasks Structure made up a larger proportion for the intended SCP groups $(\sim 23 \%)$ than for the intended PCP groups ( $\sim 15 \%)$, accounted for by the prevalence of the theme "ineffective/incorrect/missing templates for consult request by PCP" among the SCP groups' responses. When asked what can be improved, one SCP answered,

Revise the templates. Formulate templates as a question; document and provide associated history and physical examination documentation. Increase bar of what is expected, but decrease all the unnecessary template documentation. Set up templates that are more functional and provide an easy way to document.

Recurring themes across the seven topic areas indicate that front-line providers are concerned about infrastructure that facilitates, or hinders, the relationships between providers and the communication with patients. Themes belonging to the topics Tasks Structure, Roles Clarity, Infrastructure, and 
Workload all speak of the importance of developing the task definition and technology support to coordinate care between SC and PC. Themes in the topic of Communication point to the importance of relationships between providers. Themes in the topics of Access and Patients demonstrate the need for improved mechanisms for communicating with patients.

\section{Discussion}

This nationwide focus group study of the interaction between SC and PC shows that: 1) SCPs and PCPs perceive relationship building between the two sides to be the most notable facilitator of effective communication with one another; and that 2) perceptions differ mainly in i) SCPs voicing more frequently the need for better consult request templates to be used by PCPs, and in ii) PCPs voicing more frequently the need for better access to, feedback from, and service/ coordination agreements with SCPs. Findings from this study provide a solid baseline assessment of enablers and barriers to coordination, as well as identification of existing and potential coordinating mechanisms. We frame these findings with the concepts of the AHRQ's conceptual framework for studying care coordination in health care systems (Figure 1).

\section{Baseline assessment}

The need for coordination (I.a) is shown by the recurring themes (Tables 1 and 2), which demonstrate the need to improve communication about explicit expectations for the consult request, establish clear responsibilities for the SCP and the PCP, and use standardized tools to share data needed by both sides. Although VHA has a long-standing history of using EHRs, the fact that 21 of the 24 sites identified "ineffective/incorrect/missing templates for consult request by PCP" as an improvement opportunity (Table 2 ) reveals that additional enabling resources (I.c) are necessary to fulfill the information requirements (I.e) that must be met to successfully deliver coordinated care.

VHA's EHR system currently has the ability to create a template with spaces that can be labeled for manual completion, but providers have reported a preference for structured data over free text. ${ }^{19}$ An enhanced template should therefore require minimal manual insertion of data, supporting automatic population of standard data elements (eg, routine laboratory results) wherever possible. Standardizing data elements and how they are referred to in databases across all VHA facilities is also critical for smooth coordination among providers at different facilities delivering care to a patient.

\section{Coordinating mechanisms}

Coordination processes (II.c) concerning the consult request were found to need most refinement. VHA currently uses care coordination agreements to guide consults with the intent of outlining the responsibilities of the requesting PCP and the SCP providing the consult. Expectations with regard to when to refer and what information to exchange, however, are not well understood simply from documented agreements; 20 of the 24 sites identified "noneffective service/ coordination agreements" as an improvement opportunity. Using a specific hypothetical patient case to assist clinicians in collaboratively developing and mutually agreeing upon decision rules, as suggested by Diamantidis et al, ${ }^{20}$ may provide a deeper understanding of expectations on both sides. It is important to also note that established expectations require a process for ongoing maintenance to stay current with changes in care standards and practices.

Notably, fostering underlying relationships between the SCP and the PCP was identified to be indispensable for effective coordination. Close to $5 \%$ of all the responses were under the theme "building relationships improves the work," addressed at 22 of the 24 sites. Participants often relayed accounts of how having face-to-face meetings, calling each other on the phone, or visiting each other's clinics contributed to building relationships that produced meaningful and lasting effects, outweighing the technical benefits of solely relying on EHR for information exchange. They emphasized the underlying mutual respect that can then lead to a shared understanding of expectations and improved relational coordination (II.a), upon which agreements should be built.

\section{Limitations and future work}

The items mentioned earlier in this section, in the context of baseline assessment and coordinating mechanisms, clearly point to the need to design or redesign several tools and processes to enhance care coordination between the SCP and the PCP. These include 1) creating opportunities for providers to develop an understanding of (and exchange expectations regarding) each other's work, 2) collaboratively developing care coordination agreements that specify roles and responsibilities of each side, 3) standardizing information that should be shared (if using EHR, the system should support automatically populating templates to minimize error and missing information), 4) using hypothetical patients to test out the usefulness of the developed agreements, and 5) clarifying a process for ongoing maintenance of the agreements as expectations and available technology change through time. In relation to the representative themes discussed earlier in this section for baseline assessment and coordinating mechanisms, Strategies 1 and 2 respond to the theme "building relationships improves the work," Strategy 3 responds 
to the theme "ineffective/incorrect/missing templates for consult request by PCP," Strategy 4 responds to the theme "noneffective service/coordination agreements," and Strategy 5 ensures a continued responsiveness to all three themes.

The importance of professional socialization in building collaborative work relationships has been suggested within PC in the context of patient-centered medical homes (PCMHs), ${ }^{21}$ and warrants investigation regarding its usefulness in opportunities for building SCP-PCP relationships. Greenberg et al extend the PCMH concept to SC as well in their medical neighborhood framework, emphasizing the need for these neighborhoods to be built upon effective collaborative care agreements between the disciplines. ${ }^{22}$ These agreements can specify what information should be shared across the disciplines, and knowledge regarding how to best capture this information in EHRs is expected to become available following ongoing systematic reviews to understand how EHR should be structured. ${ }^{23}$ EHR templates, in addition to care agreements, can also be tested out on hypothetical patients to assess further improvements needed; use of hypothetical patients continues to serve as an effective close-to-real-world and without-risk-to-patient approach to trialing newly developed health care delivery processes. ${ }^{24}$ Sustaining the benefits of adopted processes is a challenging task for any implementation effort, ${ }^{25}$ which makes it necessary to keep these agreements and templates up to date in order to ensure that they continue to be meaningful to the providers using them.

Recognizing this study's ability to uncover these clear next steps also reveals its limitations. Originally designed to support VHA's operational improvement objectives more directly than its research goals, study resources were prioritized neither toward collecting detailed numbers and characteristics of the focus group participants nor toward strictly prohibiting the small number of cases in which an SCP focus group was attended by a PCP (or vice versa). The use of focus groups instead of individual interviews with the providers may have resulted in group dynamics affecting the responses collected, despite focus groups' strength of allowing participants' thoughts and conversations to be stimulated by one another, as well as the groups being run by trained moderators who were experienced in eliciting opinions from all individuals in a group setting. Furthermore, session moderators were not explicitly instructed to identify the participant offering each scribed response, which makes difficult further analysis of the collected data to identify whether perceptions differ by participant characteristics within SC (or PC) groups.
The questions asked of the focus group participants align most closely with the concepts of information requirements and relational coordination from the AHRQ's conceptual framework (Figure 1), yet seek open-ended information on interaction behaviors of the providers that can be improved, rather than remaining within boundaries of any particular conceptual framework. The questions therefore did not explicitly ask, for example, who in the organization would be able to lead these next steps, nor how and whether staff can be regrouped to better facilitate the needed coordination. Our decision to frame the results using the AHRQ's conceptual framework (Figure 1) allows us to specify that these missing pieces belong under the framework's concepts of predisposing characteristics (I.b) and organizational design options (II.b), and that they require careful attention as they are likely to significantly differ across different networks, facilities, and even individual clinics.

These limitations can be addressed through carefully designed future research focusing on organizational characteristics that influence care coordination, and can be run in parallel with an evaluation of pre-change current-state outcomes (ie, outcomes tied to the baseline assessment and coordinating mechanisms identified through this focus group study). For components of this research that will put grounded thematic analysis to use, incorporating the Heideggerian approach $^{26}$ could help foster a deeper understanding of the constitutive meaning of themes that emerge from the analysis. In its 2007 report, the AHRQ emphasizes the need for these outcomes, which make up the remaining third of its conceptual framework (Figure 1, III), to be centered on both patient outcomes and cost outcomes. ${ }^{9}$ AHRQ's updated Care Coordination Measures Atlas, ${ }^{8}$ especially following its recent detailed application to a systematic review of care coordination measurements, ${ }^{27}$ can be a useful guide in conceptualizing the outcomes measurements to be carried out. And once an instrument for collecting this outcomes data is either adopted from previous work ${ }^{28-31}$ or built, it can also be used for evaluating post-change future state outcomes, once the next steps listed above have been implemented to enhance care coordination.

Future work can improve upon our methodological approach by 1) reflecting an operational framework of care coordination from early on in the study planning process, 2) rigorously applying participant inclusion criteria and collecting detailed data on participant characteristics, and 3) allowing opportunities for participants (including patients and/or their family members, as well as administrators and other health system representatives, in addition 
to front-line providers) to voice their concerns in a more confidential manner. These potential methodological improvements should certainly be designed with ample consideration given to how their incorporation may affect the broad reach and operational relevance of conducting the study, which are our study's strong attributes that come from being originally initiated by VHA as a quality improvement effort.

\section{Conclusion}

This study identifies concrete tools and processes for developing the tools (eg, collaboratively developing care coordination agreements and setting up processes to continuously monitor these agreements to reflect latest regulations and technology) that medical facilities can incorporate into their practices for improved care coordination between SCPs and PCPs, based on its finding that relationships between the two sides are what fundamentally facilitate effective coordination, and specifically that providers regard both formal and informal relationship-building opportunities to be essential for building these relationships that positively impact their coordination work; medical records, consult requests, and other documented agreements can serve their full purpose of information-sharing if designed with collaborative input from both sides. Moreover, these coordination issues are found to be prevalent even within the integrated system of care that VHA's facilities offer, and can therefore be expected to apply to other non-VHA facilities facing coordination challenges.

\section{Acknowledgments}

The authors would like to thank Cliona Archambeault and Dr James Schlosser for their generous input and comments, Rael Elstein and Anuradha Ramanath for their contributions to the thematic coding, Jenniffer Leyson for her guidance on qualitative analysis, as well as the moderators, facility points-of-contact, and focus group participants for their involvement in the study.

The views expressed in this article are those of the authors and do not necessarily reflect the position or policy of the Department of Veterans Affairs or the United States Government.

\section{Disclosure}

The authors report no conflicts of interest in this work.

\section{References}

1. Forrest CB, Glade GB, Baker AE, Bocian A, von Schrader S, Starfield B. Coordination of specialty referrals and physician satisfaction with referral care. Arch Pediatr Adolesc Med. 2000;154:499-506.
2. McLeod J, McMurray J, Walker JD, Heckman GA, Stolee P. Care transitions for older adults with musculoskeletal disorders: continuity from the providers' perspective. Int J Integr Care. 2011;11:e014.

3. Pronovost PJ, Thompson DA, Holzmueller CG, et al. Toward learning from patient safety reporting systems. J Crit Care. 2006;21:305-315.

4. Suresh G, Horbar JD, Plsek P, et al. Voluntary anonymous reporting of medical errors for neonatal intensive care. Pediatrics. 2004;113: 1609-1618.

5. Toscan J, Mairs K, Hinton S, Stolee P. Integrated transitional care: patient, informal caregiver and health care provider perspectives on care transitions for older persons with hip fractures. Int J Integr Care. 2012;12:1-14.

6. Koopman RJ, Kochendorfer KM, Moore JL, Mehr DR, Wakefield S. A diabetes dashboard and physician efficiency and accuracy in accessing data needed for high-quality diabetes care. Ann Fam Med. 2010;9: 398-405.

7. Jiwa M, Walters S, Mathers N. Referral letters to colorectal surgeons: the impact of peer-mediated feedback. Br J Gen Pract. 2004;54: $123-126$.

8. Agency for Healthcare Research and Quality. Care Coordination Measures Atlas Update. Rockville, MD: Agency for Healthcare Research and Quality; 2014. Available from: http://www.ahrq.gov/ professionals/prevention-chronic-care/improve/coordination/atlas2014/ index.html. Accessed November 23, 2014.

9. McDonald KM, Sundaram V, Bravata DM, et al. Closing the Quality Gap: A Critical Analysis of Quality Improvement Strategies. Technical Review 9. Volume 7 - Care Coordination. Rockville, MD: Agency for Healthcare Research and Quality; 2007.

10. Andersen RM. Revisiting the behavioral model and access to medical care: does it matter? J Health Soc Behav. 1995;36:1-10.

11. Donabedian A. The Criteria and Standards of Quality. Ann Arbor, MI: Health Administration Press; 1982.

12. Donabedian A. Evaluating the quality of medical care. 1966. Milbank $Q$. 2005;83:691-729.

13. Nadler D, Tushman M. Strategic Organization Design. Glenview, IL and London, England: Scott, Foresman and Company; 1988.

14. Gittell JH, Fairfield KM, Bierbaum B, et al. Impact of relational coordination on quality of care, postoperative pain and functioning, and length of stay: a nine-hospital study of surgical patients. Med Care. 2000;38:807-819.

15. Miles MB, Huberman AM. Qualitative Data Analysis. 2nd ed. Thousand Oaks, CA: Sage; 1994.

16. VHA Handbook 1058.05. Washington, DC: Department of Veterans Affairs, Veterans Health Administration; 2011. Available from: http:// www.va.gov/vhapublications/ViewPublication.asp?pub_ID=2456. Accessed November 23, 2014.

17. Institute for Healthcare Improvement. The Breakthrough Series: IHI's Collaborative Model for Achieving Breakthrough Improvement. IHI Innovation Series white paper. Boston, MA: Institute for Healthcare Improvement; 2003. Available from: http://www.IHI.org. Accessed November 23, 2014.

18. Stockdale M. Analyzing Focus Group Data with Spreadsheets. American Journal of Health Studies; 2002.

19. Thong JF, Mok P, Loke D. A quality assurance survey to improve communication between ENT specialists and general practitioners. Singapore Med J. 2010;51:796-799.

20. Diamantidis CJ, Powe NR, Jaar BG, Greer RC, Troll MU, Boulware LB. Primary care - specialty care collaboration in the care of patients with chronic kidney disease. Clin J Am Soc Nephrol. 2011;6:334-343.

21. Miller WL, Cohen-Katz J. Creating collaborative learning environments for transforming primary care practices now. Fam Syst Health. 2010;28:334-347.

22. Greenberg JO, Barnett ML, Spinks MA, Dudley JC, Frolkis JP. The "medical neighborhood": integrating primary and specialty care for ambulatory patients. JAMA Intern Med. 2014;174:454-457.

23. Hyppönen H, Saranto K, Vuokko R, et al. Impacts of structuring the electronic health record: a systematic review protocol and results of previous reviews. Int J Med Inform. 2014;83:159-169. 
24. Kishi Y, Kurosawa H, Horikawa N, Hatta K, Meller W. Diagnoses of psychiatric disorders in hypothetical patients by non-psychiatric physicians in Japan. Int J Psychiatry Med. 2014;47:65-74.

25. Doyle C, Howe C, Wookcock T, et al. Making change last: applying the NHS institute for innovation and improvement sustainability model to healthcare improvement. Implement Sci. 2013;8:127.

26. Wilson HS, Hutchinson SA. Triangulation of qualitative methods: Heideggerian hermeneutics and grounded theory. Qual Health Res. 1991;1:263-276.

27. Schultz EM, Pineda N, Lonhart J, Davies SM, McDonald KM. A systematic review of the care coordination measurement landscape. BMC Health Serv Res. 2013;13:119.
28. Bonomi AE, Wagner EH, Glasgow RE, VonKorff M. Assessment of chronic illness care (ACIC): a practical tool to measure quality improvement. Health Serv Res. 2002;37:791-820.

29. Grimmer K, Moss J. The development, validity and application of a new instrument to assess the quality of discharge planning activities from the community perspective. Int J Qual Health Care. 2001;13:109-116.

30. Starfield B, Steinwachs D, Morris I, Bause G, Siebert S, Westin C. Concordance between medical records and observations regarding information on coordination of care. Med Care. 1979;17:758-766.

31. Temkin-Greener H, Gross D, Kunitz SJ, Mukamel D. Measuring interdisciplinary team performance in a long-term care setting. Med Care. 2004;42:472-481. 


\section{Supplementary materials \\ Number of responses for all themes \\ Communication}

Themes related to relationships, training, and communication between specialty care (SC) and primary care (PC) provider (SCP and PCP) teams ( $29 \%$ of total number of responses)

Table SI Communication themes

\begin{tabular}{ll}
\hline & Number of responses \\
\hline Strong Practice theme & 120 \\
Building relationships improves the work & 59 \\
Informal consults are appreciated & 42 \\
Interdisciplinary meetings help & 41 \\
Easy to contact SCP & 26 \\
Feedback is important & 23 \\
Easy to contact PCP & 18 \\
PCP learns from each consult & 8 \\
Outlook communication is helpful & 337 \\
Total & \\
Improvement Opportunity theme & 80 \\
Lack of feedback & 77 \\
Difficulty contacting SCP team & 58 \\
Structured PCP training recommended & 47 \\
Lacking relationships & 40 \\
Difficulty contacting PC team & 16 \\
Lack of PCP knowledge & 14 \\
Disagreements occur over patient care plan & 14 \\
Specialty-to-specialty teamwork recommended & 14 \\
Outlook communication problematic & 11 \\
Unofficial communication too time-consuming & 5 \\
Delayed results/feedback & 365 \\
Total & \\
\hline Abbreviations: SCP, spera & \\
\hline
\end{tabular}

Abbreviations: SCP, specialty care provider; $\mathrm{PCP}$, primary care provider; $\mathrm{PC}$ primary care.

Table S2 Tasks structure themes

\begin{tabular}{|c|c|}
\hline & $\begin{array}{l}\text { Number of } \\
\text { responses }\end{array}$ \\
\hline \multicolumn{2}{|l|}{ Strong Practice theme } \\
\hline Effective/correct templates for consult request by PCP & 36 \\
\hline Cosigning PCP on notes is helpful & 35 \\
\hline Total & 71 \\
\hline \multicolumn{2}{|l|}{ Improvement Opportunity theme } \\
\hline $\begin{array}{l}\text { Ineffective/incorrect/missing templates for consult request } \\
\text { by PCP }\end{array}$ & 90 \\
\hline Incorrect/insufficient administrative practice & 55 \\
\hline PCP needs clear plan, guidelines for f/up & 49 \\
\hline Consult prework too difficult/time-consuming & 39 \\
\hline Incorrect/insufficient medical information provided to SCP & 35 \\
\hline Too much documentation work & 28 \\
\hline Priority classification improvements needed & 24 \\
\hline Insufficient/improper medical examination/practice by PCP & 23 \\
\hline Unnecessary consults & 22 \\
\hline E-consult availability needed & 22 \\
\hline Reminder-induced consults create unwanted visits & 5 \\
\hline Unnecessary f2f consult & 2 \\
\hline Total & 394 \\
\hline
\end{tabular}

Abbreviations: SCP, specialty care provider; PCP, primary care provider; f/up, follow-up; f2f, face-to-face.

\section{Tasks structure}

Themes related to task guidelines and standards (ie, what is expected to be done) ( $\sim 19 \%$ of total number of responses)

Table S3 Patient perception themes

\begin{tabular}{ll}
\hline & Number of responses \\
\hline Strong Practice theme & \\
Emphasis on communicating with patients & 57 \\
Perception of good care & 33 \\
Total & 90 \\
Improvement Opportunity theme & \\
Lack of care coordination & 91 \\
Gaps in communication with patient & 67 \\
Patients don't understand process or roles & 38 \\
Dual care complicates & 25 \\
Problems related to no shows & 12 \\
Patient concern for PCP knowledge & 6 \\
of SCP work & \\
Copay problems & 4 \\
Noncompliant patients & 3 \\
Total & 246 \\
\hline
\end{tabular}

Abbreviations: SCP, specialty care provider; $\mathrm{PCP}$, primary care provider.

\section{Patients}

Themes related to patient perceptions, quality of care for patients, and patient characteristics $(\sim 14 \%$ of total number of responses)

Table S4 Organizational role themes

Number of responses

Strong Practice theme

Effective service/coordination agreements $\quad 34$

PC RN care manager role importance 2I

Work together to see reluctant patients 3

Total 58

Improvement Opportunity theme

Noneffective service/coordination agreements 53

Fee basis complexities or under-use 31

Complexity related to facility-to-facility 29

differences

Central or direct scheduling recommended 23

Unknown contact in PC or SC 19

Too much handed back to PCP from referral 19

$\begin{array}{ll}\text { Varied methods of graduation } & 17\end{array}$

Shared reminder responsibility and clarity $\quad 16$

needed

SCP clinician limitations $\quad 16$

Veteran travel scheduling gaps 9

Untracked consults 6

PCP should always refer 5

Unutilized cancellations 3

SC should refer if urgent 2

SC recommends plan not referred for by PC I

Total 249

Abbreviations: SCP, specialty care provider; PCP, primary care provider; PC primary care; SC, specialty care; RN, Registered nurse. 


\section{Roles clarity}

Themes related to organizational roles (ie, who is responsible for what) ( $13 \%$ of total number of responses)

Table S5 Geographical distance themes

Number of responses

\section{Strong Practice theme}

Information systems facilitate care

40

Nearby SCP facilitates effective care

Total

13

53

Improvement Opportunity theme

CPRS limitations

71

Distance technology solutions desirable $\quad 34$

Distant SCP creates challenges $\quad 30$

Clinical space limitations exist 20

Lack of visibility 13

Slow computing 6

No secure messaging 3

Total 177

Abbreviations: SCP, specialty care provider; CPRS, computerized patient record system.

\section{Infrastructure}

Themes related to geographical distances, outpatient clinics, space, and information systems and technology ( $\sim 9 \%$ of total number of responses)

Table S6 Supply/staffing and workload balance themes

\begin{tabular}{ll}
\hline & Number of responses \\
\hline Improvement Opportunity theme & \\
More support staff needed & 56 \\
Not enough specialists & 44 \\
Student continuity issues & 43 \\
Turnover (PCP/SCP/staff) & 18 \\
PCP workload too high & 18 \\
Split time/part time complexities & 17 \\
Recommend NP staffing & 13 \\
Burnout & 3 \\
Total & 212 \\
\hline
\end{tabular}

Abbreviations: $\mathrm{SCP}$, specialty care provider; $\mathrm{PCP}$, primary care provider; $\mathrm{NP}$, nurse practitioner.

\section{Workload}

Themes related to supply/staffing and workload balance ( $9 \%$ of total number of responses)

\section{Access}

Themes related to access for patient appointments and availability to reach SCPs/PCPs on the phone ( $\sim 7 \%$ of total number of responses)

Table S7 Access themes

\begin{tabular}{ll}
\hline & Number of responses \\
\hline Strong Practice theme & \\
Timeliness of consults to appt is good & 24 \\
SCP at CBOC improves access & 5 \\
Total & 29 \\
Improvement Opportunity theme & \\
Poor SCP access for patients & 52 \\
Poor PCP access for patients & 25 \\
Insufficient timeliness of consults/appts & 24 \\
Poor phone systems & 20 \\
SCP not where need is (eg, CBOC) & 9 \\
Short appts & 2 \\
Total & 132 \\
\hline
\end{tabular}

Abbreviations: appt, appointment; SCP, specialty care provider; $\mathrm{PCP}$, primary care provider; $\mathrm{CBOC}$, community-based outpatient clinic.
Journal of Multidisciplinary Healthcare

\section{Publish your work in this journal}

The Journal of Multidisciplinary Healthcare is an international, peerreviewed open-access journal that aims to represent and publish research in healthcare areas delivered by practitioners of different disciplines. This includes studies and reviews conducted by multidisciplinary teams as well as research which evaluates the results or conduct of such teams or

\section{Dovepress}

healthcare processes in general. The journal covers a wide range of areas and welcomes submissions from practitioners at all levels, from all over the world. The manuscript management system is completely online and includes a very quick and fair peer-review system. Visit http://www.dovepress.com/testimonials.php to read real quotes from published authors. 